\title{
SHARING RISK - AN ECONOMIC PERSPECTIVE
}

BY

Andreas KuLl

\begin{abstract}
We revisit the relative retention problem originally introduced by de Finetti using concepts recently developed in risk theory and quantitative risk management. Instead of using the Variance as a risk measure we consider the Expected Shortfall (Tail-Value-at-Risk) and include capital costs and take constraints on risk capital into account. Starting from a risk-based capital allocation, the paper presents an optimization scheme for sharing risk in a multi-risk class environment. Risk sharing takes place between two portfolios and the pricing of risktransfer reflects both portfolio structures. This allows us to shed more light on the question of how optimal risk sharing is characterized in a situation where risk transfer takes place between parties employing similar risk and performance measures. Recent developments in the regulatory domain ('risk-based supervision') pushing for common, insurance industry-wide risk measures underline the importance of this question. The paper includes a simple nonlife insurance example illustrating optimal risk transfer in terms of retentions of common reinsurance structures.
\end{abstract}

\section{KEYWORDS}

Sharing and pooling of risk, reinsurance, optimal retentions, risk-based capital, capital cost

\section{INTRODUCTION}

Actuarial and financial risk management has evolved rapidly over the last couple of years. Driving forces behind this development are manyfold. To name a few: Increasing integration of financial and actuarial risk management, a shift towards performance and risk management from an economical perspective and recent pressure on profitability in the insurance industry. Considerable theoretical progress has been achieved, an example of this being the development of coherent risk measures. Further, current developments in the regulatory domain will eventually lead to a supervisory framework in which insurers (and reinsures) will have to quantify risk-based capital using prescribed risk measures (see e.g. [22, 12]. 
Given this background, we revisit the relative retention problem originally introduced by de Finetti [10] from a more modern point of view. Instead of using Variance as a risk measure we consider the Expected Shortfall (TailValue-at-Risk) and include capital costs and constraints on risk-based capital into our considerations. Taking into account constraints on risk-based capital means that capital restrictions are met. These constraints are of growing importance from a risk management and regulatory point of view. Finally, considering capital costs is of importance when it comes to deciding on how much risk should be kept on the balance sheet from an economical perspective. The basic question to answer then becomes: Is it economically favorable to support risk by capital or should a transfer of risk take place, for example by buying reinsurance? And, in this respect, what are the optimal risk retentions of a reinsurance program?

Setting retention levels of reinsurance programs has been discussed by many authors. Starting with de Finetti [10], there has been a long history of attempts to set retention levels in such a way that given a constraint on the net profit, the overall Variance of the portfolio is minimized ([23, 3, 4, 26, 19, 20, 15]). Other approaches rely on criteria related to adjustment coefficients $[5,7]$ or utility theory [6]. For a comprehensive review see [8]. A limitation of most of those approaches is that they focus on the primary insurer while the point of view of the reinsurer is taken into account only by simplified premium principles, often limited to the expected value or variance premium principle, i.e. premium principles that rely on first or second moments of loss distributions.

The paper is organized as follows. In the next Section we briefly discuss some mathematical concepts and tools that will be used in the paper. The third Section derives conditions for optimal relative retentions and discusses the question of setting absolute retention levels. Finally, Section four presents a simple example. The paper concludes with a summary and a discussion.

\section{From Risk EXPOSURE TO THE MEASUREMENT AND THE PRICE OF RISK}

\subsection{Risk measures}

In insurance and finance, risk refers to the intrinsic uncertainty of the future and its impact on business goals. Mathematically, uncertainty can be described by a random variable $X$, viewed as an element of a linear space of measurable functions $\mathcal{L}$ and defined on an appropriate probability space. A risk measure for $X$ then is defined by a reasonable map $\rho: F_{X} \mapsto \mathbb{R}$ mapping $X$ or the cumulative distribution $F_{X}$, respectively, to a single, risk quantifying number.

A basic example for a risk measure is the Variance of $X$. It measures the variability of $X$ relative to the expectation $\mathrm{E}[X]$, however, it falls short of characterizing the tail of the distribution. A common risk measure characterizing the tail of a distribution is the Value-at-Risk. It is defined as the $\alpha$-quantile $F_{X}^{-1}(\alpha)$ and denoted by $\operatorname{VaR}_{\alpha}$. $\operatorname{VaR}$ as risk measure is very convenient in the 
sense that it has the a straightforward interpretation as the impact of an event with given probability $\alpha$, however it does not distinguish between distributions having equal $\alpha$-quantiles but different tails beyond. A risk measure including this type of information is the Expected Shortfall, defined as conditional average

$$
\mathrm{ES}_{\alpha}[X]=E\left[X \mid X \leq F_{X}^{-1}(\alpha)\right]
$$

where it is assumed that smaller realizations $x$ correspond to an adverse situation ${ }^{1}$. This is typical the case if $X$ stands for a profit and loss distribution. The Expected Shortfall quantifies in this case the risk related to events falling short of $\operatorname{VaR}_{\alpha}=F_{X}^{-1}(\alpha)$ as the average of all outcomes lower than $\operatorname{VaR}_{\alpha}$, or, in a discrete Monte Carlo setting as average over a subset of scenarios (see [1]). As discussed in [2], the Expected Shortfall is coherent and possesses desirable properties like

$$
\begin{aligned}
\mathrm{ES}_{\alpha}[X+Y] & \geq \mathrm{ES}_{\alpha}[X]+\mathrm{ES}_{\alpha}[Y] \text { sub-additivity } \\
\mathrm{ES}_{\alpha}[X] & \leq \mathrm{ES}_{\alpha}[Y], \text { if } X \leq Y \text { monotonicity } \\
\mathrm{ES}_{\alpha}[s \cdot X] & =s \cdot \mathrm{ES}_{\alpha}[X] \text { homogeneity } \\
\mathrm{ES}_{\alpha}[X+a] & =\mathrm{ES}_{\alpha}[X]+a \text { translation invariance }
\end{aligned}
$$

While the coherence of the risk measure is not a prerequisite for what follows we will consider the Expected Shortfall as risk measure because of its stability (see e.g. [18] and comments in Section 3.5.1) and the fact that it is the risk measure that has been chosen for a well developed risk-based supervisory framework (Swiss Solvency Test, see e.g. [22]).

\subsection{Risk-based capital and cost of capital}

At company level, capital provides protection and mitigation from insolvencyor ruin due to large unexpected losses. Holding such 'risk capital' comes at a cost since shareholders expect an adequate return from their investment. Any economically meaningful analysis of insurance performance should take this cost of capital into account.

In the following we will distinguish between risk capital and risk-based capital (RBC). With risk-based capital we denote capital that is allocated to support specific risk(s) while risk capital stands for the available amount of capital to support risk in total. The definition and allocation of risk-based

For our purposes $F$ may further depend on a parameter $r$ and $F(r)$ is assumed to be piecewise continuous or approximated by a continuous function. The definition of the Expected Shortfall as an average (or integral) over the tail of the distribution $F$ is important as it will allow for partial derivatives $\partial_{r} \operatorname{ES}[F(r)]$ to exist (see Section 3.3). 
capital is a widely discussed problem and ranges from simple factor based approaches used for regulatory purposes (e.g. Solvency I) to more elaborated concepts relying on conditional risk measures or distorted probabilities. Riskbased capital in our context refers to company internal capital management but can also have a link to regulatory capital requirements (e.g. as constraints due to capital requirements in a risk-based supervision regime). Independent of the definition and allocation of risk-based capital, the cost of capital $C$ related to a specific risk is proportional to the amount of allocated risk-based capital, i.e.

$$
C=\lambda \cdot \mathrm{RBC}
$$

The cost of capital rate $\lambda$ depends on various factors including e.g. the capital structure of the company, type of business and other features. Frequently, the CAPM model is used to estimate $\lambda^{2}$.

We will now consider the simplified situation of a non-life insurer that underwrites one short-tail line of business (i.e. business for which risk materializes over a relatively short time horizon) over a single time period and is ceding part of the associated risk to a reinsurer. Neglecting expenses, discounting and the cost of allocated capital, the net underwriting profit is

$$
U=P-L-P^{*}+R
$$

where $P$ stands for the gross premium income of the primary insurer, $P^{*}$ for paid reinsurance premiums, $L$ for the accumulated gross loss amount and $R$ for the accumulated recoveries due to reinsurance. We will not consider loss dependent, stochastic premiums here. Thus the basic accumulated stochastic variable is the gross loss amount $L$, which may include several risk classes $i$

$$
L=\sum_{i} \sum_{j=1}^{N_{i}} l_{i, j}
$$

with stochastic loss amounts $l_{i, j}$ and the stochastic number of losses $N_{i}$. Recoveries $R$ will depend on the loss experience and some control parameters $r$ (e.g. retentions), i.e. $R=R\left(r_{i}, l_{i, j} \mid i, j\right)$. Including the cost of capital, the net underwriting profit $Z$ including capital costs becomes

$$
Z=U-\lambda \cdot \mathrm{RBC}
$$

where RBC stands for the capital allocated to support the net risk. This framework can easily be generalized to include discounting, investment return and risk, expenses and typical features of non-life insurance like loss depended premiums or profit sharing.

2 The cost of capital rate $\lambda$ for an insurer typically lies in the range $4 \%$ to $15 \%$. 
Insurers normally do not allocated risk-based capital for the full range of potential adverse results but only up a probability corresponding to a company specific risk appetite level $\alpha$ or up to regulatory requirements ${ }^{3}$. Equally important, the determination of risk-based capital also depends on the risk measure and other factors. For example, the risk-based capital can be defined relative to the break-even situation $z \in\{Z=0\}$ or relative to the expected profit $z \in$ $\{Z=\mathrm{E}[Z]\}$. Note that from a theoretical point of view there are properties that favor some of the concepts over other ones. This is in particular the case for a risk-based capital allocation on sub-portfolio level (see e.g. [24, 25] and Section 2.3). We here choose the Expected Shortfall as risk measure and define risk-based capital relative to the break-even situation $z \in\{Z=0\}$. The risk-based capital for a given risk appetite level $\alpha$ then is determined implicitly by

$$
\begin{aligned}
\mathrm{RBC}[Z] & =-\mathrm{ES}_{\alpha}[Z] \\
& =-\mathrm{ES}_{\alpha}[U-\lambda \cdot \operatorname{RBC}[Z]]
\end{aligned}
$$

Using (2.5) and solving this equation for $\mathrm{RBC}[Z]$ we get

$$
\operatorname{RBC}[Z]=-\frac{\mathrm{ES}_{\alpha}[U]}{1-\lambda}
$$

and thus

$$
Z=U+\lambda \cdot \frac{\mathrm{ES}_{\alpha}[U]}{1-\lambda}
$$

Note that the sign in (2.11) has been chosen in a way that the $\mathrm{RBC}[Z]$ is a positive number under normal circumstances. The net profit $Z$ is the basic quantity we are interested in. For the insurer, it characterizes the economical value of keeping risk on the balance sheet.

\subsection{Generalization for a portfolio of risks}

For various purposes ranging from risk management to performance measurement, insurers typically group their business into several classes ('lines of business'). The allocation of capital usually follows this grouping which leads to a multivariate situation. There is general agreement that a capital allocation scheme should fulfill two key properties:

- Risk-based capital should be allocated according to the risk contribution of the sub-portfolio to the overall risk of the portfolio.

- The sum of allocated risk-based capital should equal the risk-based capital determined for the total portfolio.

3 Depending on purposes, $\alpha$ typically lies between $99 \%$ and $99.99 \%$. 
A number of different capital allocation schemes taking into account these key properties have been proposed, many relying on conditional risk measures. For a review see [25]. In addition, there are capital allocation scheme applying a game theoretical or optimization approach (see $[9,16]$ and also comments in Sections and Conclusions).

We consider here a capital allocation based on the conditional Expected Shortfall (called also the Expected Shortfall 'co-measure' in [25]). Given a set of risk factors $\left\{X_{i}\right\}$, the conditional Expected Shortfall is defined as

$$
\mathrm{CES}_{\alpha, X}\left[X_{i}\right]:=\mathrm{E}\left[X_{i} \mid X \leq F_{X}^{-1}(\alpha)\right]
$$

which is the mean of $X_{i}$ given that the sum $X=\sum X_{i}$ is greater than or equal to the $\alpha$-quantile $F_{X}^{-1}(\alpha)$. The quantity $\mathrm{CES}_{\alpha, X}\left[X_{i}\right]$ can be interpreted as the average contribution of the sub-portfolio $i$ to the average adverse portfolio result for $X \leq F_{X}^{-1}(\alpha)^{4}$. A basic property of conditional risk measures is their additivity. In case of the conditional Expected Shortfall

$$
\operatorname{ES}_{\alpha}[X]=\operatorname{CES}_{\alpha, X}[X]=\sum_{i} \operatorname{CES}_{\alpha, X}\left[X_{i}\right]
$$

which is a consequence of the conditional nature of $\mathrm{CES}_{\alpha, X}\left[X_{i}\right]$.

A generalization of equations (2.10) to (2.12) to $n$ sub-portfolios is straightforward. The contribution of a sub-portfolio $i$ to the total risk-based capital becomes

$$
\begin{aligned}
\operatorname{RBC}\left[Z_{i}\right] & =-\mathrm{E}\left[Z_{i} \mid Z \leq F_{Z}^{-1}(\alpha)\right] \\
& =-\operatorname{CES}_{\alpha, Z}\left[Z_{i}\right] \\
& =-\operatorname{CES}_{\alpha, Z}\left[U_{i}-\lambda \cdot \operatorname{RBC}\left[Z_{i}\right]\right] \\
& =-\frac{\operatorname{CES}_{\alpha, Z}\left[U_{i}\right]}{1-\lambda}
\end{aligned}
$$

where $Z_{i}$ is the insurer's profit of the $i$-th sub-portfolio. Using the allocation scheme (2.15) for risk-based capital, the profit on sub-portfolio level is

$$
\begin{aligned}
Z_{i} & =U_{i}-\lambda \cdot \operatorname{RBC}\left[Z_{i}\right] \\
& =U_{i}+\lambda \cdot \frac{\operatorname{CES}_{\alpha, Z}\left[U_{i}\right]}{1-\lambda}
\end{aligned}
$$

4 Under the assumption of homogeneity of the function $f(r)=\operatorname{ES}_{\alpha}[X(r)]$ where $r$ is a control parameter characterizing the transferred risk $R=X(r)$, this capital allocation scheme is equivalent to the Euler allocation principle (see e.g. [24]). Homogeneity of $f(r)$ in the context of reinsurance is given for proportional reinsurance agreements where $R=(1-r) \cdot X$. For non-proportional reinsurance agreements where $R=X(r)$ is non-linear, this is however not in general the case. For a related discussion see also [17]. 
The term

$$
\lambda \cdot \frac{\mathrm{CES}_{\alpha, Z}\left[U_{i}\right]}{1-\lambda}
$$

in (2.16) corresponds to capital costs for risk class $i$ (after reinsurance). Capital costs depend on the portfolio structure of the insurer and thus take into account diversification effects. In other terminology, (2.17) quantifies the (net) loading related to risk class $i$.

The total expected underwriting result after capital costs simply is

$$
\mathrm{E}[Z]=\sum_{i} \mathrm{E}\left[Z_{i}\right]
$$

Due to the additivity of the conditional Expected Shortfall we get a similar expression for the total risk at company level by summing over the contributions of the sub-portfolios

$$
\mathrm{ES}_{\alpha}[Z]=\sum_{i} \mathrm{CES}_{\alpha, Z}\left[Z_{i}\right]
$$

and the risk-based capital exhibits the similar additivity

$$
\mathrm{RBC}[Z]=\sum_{i} \mathrm{RBC}\left[Z_{i}\right]=-\sum_{i} \frac{\mathrm{CES}_{\alpha, Z}\left[U_{i}\right]}{1-\lambda}
$$

This demonstrates that (i) the risk-based capital allocation to lines of business depends on the risk characteristic on sub-portfolio level and (ii) the risk-based capital of the $n$ sub-lines adds up to the total risk-based capital at company level. Note that $-\sum_{i} \mathrm{CES}_{\alpha, Z}\left[Z_{i}\right] \leq-\sum_{i} \mathrm{ES}_{\alpha}\left[Z_{i}\right]$ (see e.g. [14] for details). The allocation scheme thus takes into account diversification on portfolio level and allocates the diversification benefit on portfolio level to the sub-portfolio level. It follows directly that capital costs on sub-portfolio level share properties (i) and (ii) as well.

\section{OptIMAL RISK RETENTION}

\subsection{Sharing and transferring risk}

As discussed, keeping risk on a balance sheet comes at a cost for an insurance company. Pooling and sharing risk will in general lower this cost due to diversification effects. The insurance industry achieves intercompany risk sharing by risk transfer commonly relying on reinsurance. Because of the usually higher diversification of the portfolio of the reinsurer, the reinsurance premium for risk transfer may be lower than the corresponding cost of keeping the risk on the balance sheet of the insurer only. Assuming that a reinsurer gets an 
adequate premium, risk transfer can thus lead to a situation where both the risk ceding and receiving party benefit. The basic question thus arising is how and to what extend risk should be retained or shared. Answering this question depends on risk characteristics and a number of other parameters including e.g. the type of risk measure, the risk appetite level and capital costs. An equally important role play constraints on available risk capital.

\subsection{The price of transferring risk}

\subsubsection{Reinsurance premium principle}

Reinsurance premiums principle usually split the reinsurance premium into the expected loss and a loading. Common loadings principles are e.g. the Expected Value principle or the Variance principle (see e.g. [11]). We chose here to define the loading from a more economic point of view and consider that both the reinsurer and the primary insurer have to support underwritten risks by capital. We account for related capital costs of the reinsurer in exactly the same way as proposed in Section 2.2 for the primary insurer.

\subsubsection{Non-proportional reinsurance}

Following Section 2.2, we start from the underwriting profit distribution including the cost of capital and denote quantities referring to the reinsurer by a $\operatorname{star}(*) . Z^{*}$, the overall underwriting profit including cost of capital of the reinsurer, is

$$
Z^{*}=Z_{0}^{*}+\sum_{i} Z_{i}^{*}=Z_{0}^{*}+\sum_{i} P_{i}^{*}-R_{i}-C_{i}^{*}
$$

where $Z_{0}^{*}$ stands for the profit distribution of the reinsurance portfolio before risk transfer and $Z_{i}^{*}$ for the profit distribution related to the $i$-th risk transfer (or line of business) with reinsurance recoveries (or losses, respectively) $R_{i}$, premium income $P_{i}^{*}$ and related capital costs $C_{i}^{*}$. The cost of capital at individual risk transfer level is given by

$$
C_{i}^{*}=\lambda^{*} \cdot \mathrm{RBC}^{*}\left[Z_{i}^{*}\right]
$$

where the constant $\lambda^{*}$ stands for the capital cost of the reinsurer. In analogy to $(2.15)$ the risk-based capital $\mathrm{RBC}^{*}\left[Z_{i}^{*}\right]$ allocated to $Z_{i}^{*}$ is defined by the implicit relation

$$
\mathrm{RBC}^{*}\left[Z_{i}^{*}\right]=-\mathrm{CES}_{\beta, Z^{*}}^{*}\left[P_{i}^{*}-R_{i}-\lambda^{*} \cdot \mathrm{RBC}^{*}\left[Z_{i}^{*}\right]\right]
$$

with $\beta$ the risk appetite level of the reinsurer. Solving for $\mathrm{RBC}^{*}\left[Z_{i}^{*}\right]$ yields

$$
\operatorname{RBC}^{*}\left[Z_{i}^{*}\right]=-\frac{\mathrm{CES}_{\beta, Z^{*}}^{*}\left[P_{i}^{*}-R_{i}\right]}{1-\lambda^{*}}
$$


From equation (3.1) and expression (3.4) we define a reinsurance premium principle as follows

$$
P_{i}^{*}=\mathrm{E}\left[R_{i}\right]+\lambda^{*} \cdot \mathrm{RBC}^{*}\left[Z_{i}^{*}\right]
$$

The premium principle simply states that the reinsurance premium $P_{i}^{*}$ per risk class $i$ equals the expected accumulated loss for the reinsurer (recovery for the primary insurer) $\mathrm{E}\left[R_{i}\right]$ plus a loading $\lambda^{*} \cdot \mathrm{RBC}^{*}\left[Z_{i}^{*}\right]$ reflecting the (diversified) capital costs of the reinsurer. Solving the implicit expression (3.5) for $P^{*}$ and assuming flat reinsurance premiums (i.e. no reinstatements) leads to the reinsurance premium

$$
P_{i}^{*}=\left(1-\lambda^{*}\right) \cdot \mathrm{E}\left[R_{i}\right]+\lambda^{*} \cdot \mathrm{CES}_{\beta, Z^{*}}^{*}\left[R_{i}\right]
$$

According to (3.6) the reinsurer charges the insurer for capital costs relative to his portfolio. Risks showing a higher degree of dependency with the portfolio of the reinsurer will require a relatively higher loading than risks with no or negative dependency. In general, capital costs for reinsurer and primary insurer will differ not only because of different portfolio structures but also because $\lambda^{*} \neq \lambda$ and $\alpha \neq \beta$ i.e. the capital costs of insurer and reinsurer are not the same and the risk appetite may differ.

\subsubsection{Proportional reinsurance}

In a proportional reinsurance arrangement, primary insurer and reinsurer share both risk and premium on a proportional basis. The premium principle in the proportional case thus has the simple form

$$
P_{i}^{*}=\left(1-r_{i}\right) \cdot P_{i}
$$

where $r_{i}$ quantifies the retention of the primary insurer. In practice, a reinsurer will pay a brokerage or commission $B_{i}$ compensating acquisition expenses of the primary insurer. The discussion of an adequate reinsurance premium thus becomes a question of the adequacy of $B_{i}$. From the point of view of the primary insurer, the reinsurance premium paid for the risk transfer is

$$
P_{i}^{*}=\left(1-r_{i}\right) \cdot P_{i}-B_{i}
$$

In the following, we will assume that the reinsurer charges the primary insurer for the risk transfer by adjusting the commission $B_{i}$ and we will consider commissions $B_{i}$ only implicitly by focusing on the 'commission adjusted' reinsurance premium $P_{i}^{*}$. If needed, the commission (or brokerage) $B_{i}$ contained in $P_{i}^{*}$ can directly be recovered by solving (3.8) for $B_{i}$. 


\subsection{Criteria for optimal risk retention}

\subsubsection{Constrained risk retention}

After having fixed notation and concepts, we come back to the initial question. What are optimal risk retention levels given portfolio structure, reinsurance premium principle, and capital costs? Optimality in this perspective refers to the amount and type of risk that should be kept (or shared with the reinsurer, respectively) from an economical point of view. While this question ('absolute retention problem') certainly is relevant, it does not reflect common business realities where the amount of capital supporting risk is limited and often fixed e.g. by 'risk budgets'. We therefore take into account a constraint and consider the amount of available risk capital as fixed. According de Finetti's terminology this is the relative retention problem. Optimizing the overall level of risk retention is discussed in Section 3.3.3.

Depending on the risk characteristics of each line of business, optimal risk retentions will vary. The question to answer thus is: Given the overall risk retention in terms of the risk-based capital, what are economically optimal risk retentions $r=\left\{r_{i}\right\}$ on line of business level? In particular, given the available risk capital, how should risk be ceded? Considering the profit $Z=Z(r)$ and the risk-based capital $\mathrm{RBC}=\mathrm{RBC}[Z(r)]$ as a function of retention levels $\left\{r_{i}\right\}$, this is a typical non-linear constrained extremum value problem

$$
\begin{aligned}
\mathrm{E}[Z(r)] & =\text { max! } \\
\operatorname{RBC}[Z(r)] & =\text { const }
\end{aligned}
$$

with control parameters $\left\{r_{i}\right\}$. This type of extremum value problem is solved by Lagrangian multiplier techniques, i.e. by maximizing the expression

$$
\phi=\mathrm{E}[Z(r)]+\kappa \cdot \mathrm{RBC}[Z(r)]
$$

where $\kappa$ represents the Lagrangian multiplier associated with the constraint (3.10) given by $(2.20)^{5}$. To keep the notation simple, we will omit in the following the explicit $r$-dependence of the profits $Z, U, Z^{*}$ and $U^{*}$ as well as of the recoveries $R$, the reinsurance premium $P^{*}$ and risk-based capital $\mathrm{RBC}[Z]$ and $\mathrm{RBC}^{*}\left[Z^{*}\right]$.

Independent of the reinsurance structure, the optimization problem can be formulated in terms of the reinsurance recoveries $R_{i}$ and control parameters $r_{i}$ related e.g. to the proportional (quota share reinsurance) or non-proportional retention (excess-of-loss reinsurance). In the context of the Lagrange multiplier technique which we will make use of here, constraint optima are characterized by the condition

$$
\frac{\partial \phi}{\partial r_{j}}=0,=0, \quad \forall j
$$

5 The reverse optimization problem $\mathrm{ES}_{\alpha, Z}[Z]=\min !, \mathrm{E}[Z]=$ const leads to an expression equivalent to $(3.11)$. 
We continue by considering an explicit expression for the objective function $Z$, i.e. the net profit distribution. Combining expressions for the insurers underwriting profit $U$, the reinsurance premium $P^{*}$ and the risk-based capital $\mathrm{RBC}$ we get for $Z$

$$
\begin{aligned}
Z= & \sum_{i}\left(P_{i}-L_{i}-P_{i}^{*}+R_{i}\right)-\frac{\lambda}{1-\lambda} \cdot \mathrm{CES}_{\alpha, Z}\left[L_{i}-P_{i}+P_{i}^{*}-R_{i}\right] \\
= & \sum_{i} \frac{P_{i}}{1-\lambda}-\frac{1-\lambda^{*}}{1-\lambda} \cdot \mathrm{E}\left[R_{i}\right]-\frac{\lambda^{*}}{1-\lambda} \cdot \mathrm{CES}_{\beta, Z^{*}}^{*}\left[R_{i}\right] \\
& \quad-\left(L_{i}-R_{i}\right)-\frac{\lambda}{1-\lambda} \cdot \mathrm{CES}_{\alpha, Z}\left[L_{i}-R_{i}\right]
\end{aligned}
$$

With the corresponding expression (2.19) for the risk-based capital constraint, condition (3.12) becomes

$$
\begin{aligned}
\frac{\partial \phi}{\partial r_{j}}= & \frac{\partial}{\partial r_{j}}(\mathrm{E}[Z]+\kappa \cdot \mathrm{RBC}[Z]) \\
= & \frac{\partial}{\partial r_{j}}\left[\left(\lambda^{*}-\lambda+\kappa \cdot\left(1-\lambda^{*}\right)\right) \cdot \mathrm{E}\left[R_{j}\right]\right. \\
& \left.\quad-\sum_{i}\left[\lambda^{*} \cdot(1-\kappa) \cdot \mathrm{CES}_{\beta, Z^{*}}^{*}\left[R_{i}\right]-(\lambda-\kappa) \cdot \mathrm{CES}_{\alpha, Z}\left[R_{i}\right]\right]\right] \stackrel{!}{=} 0 \forall j
\end{aligned}
$$

Solutions $r=r_{0}$ of the constraint optimization problem (3.9) are characterized by $(3.14)^{6}$. A sufficient criteria for the existence of the maximum is the negative definiteness of the Hesse matrix $\left.\frac{\partial^{2}}{\partial r_{i} \partial r_{j}} \mathrm{E}[Z]\right|_{r=r_{0}}$ along the direction spanned by constraint (3.10) (see e.g. [21]).

What is the role and interpretation of the Lagrange multiplier $\kappa$ in (3.14)? The parameter is related in our case to the overall risk retention. The overall risk retention (corresponding to the risk-based capital amount $\mathrm{RBC}[Z]$ ) implicitly determines the Lagrangian multiplier $\kappa$. Its interpretation is linked to the derivative of the objective function $\mathrm{E}[Z]$. The value of the Lagrange multiplier $\kappa$ at a given net risk retention is equal to the derivative of $\mathrm{E}[Z]$ with respect to the retentions $r_{i}$. Thus the Lagrange multiplier $\kappa$ measures the marginal change of $\mathrm{E}[Z]$, i.e. the rate of increase (or decrease) in the maximized expected net profit $\mathrm{E}[Z]$ as retentions $r_{i}$ are changed.

Equation (3.14) form the basic criteria we will consider in detail in the following. According to (3.14), optimal retentions depend on both the primary insurer's and reinsurer's portfolio, risk measure and risk appetite and individual capital costs. These expressions thus capture the essential economics behind

6 In vector notation (3.14) can bewritten as

$$
\nabla_{r}\left[\left(\lambda^{*}-\lambda+\kappa \cdot\left(1-\lambda^{*}\right)\right) \cdot \mathrm{E}[R]-\lambda^{*} \cdot(1-\kappa) \cdot \mathrm{CES}_{\beta, Z^{*}}^{*}[R]+(\lambda-\kappa) \cdot \mathrm{CES}_{\alpha, Z}[R]\right] \stackrel{!}{=} 0
$$


risk transfer between primary insurer and reinsurer and illustrates how a 'winwin' situation can occur as well as by which factors it is driven.

As mentioned condition (3.14) is general. It does not depend on the type of risk transfer. For example, (3.14) characterizes optimal retention levels in non-proportional and proportional reinsurance agreements as well as combinations thereof.

\subsubsection{Equal portfolios and zero capital costs}

From (3.14) it follows that the difference of the portfolios of the primary insurer and the reinsurer and non-zero capital costs are crucial for the existence of a solution. To see that consider first the case where $\beta=\alpha$ and $\operatorname{CES}^{*}\left[R_{i}\right]=$ $\mathrm{CES}\left[R_{i}\right]$. Expression (3.14) becomes

$$
\frac{\partial \phi}{\partial r_{j}}=\left(\lambda^{*}-\lambda+\kappa \cdot\left(1-\lambda^{*}\right)\right) \cdot \frac{\partial}{\partial r_{j}} \sum_{i}\left[\mathrm{E}\left[R_{i}\right]-\mathrm{CES}_{\alpha, Z}\left[R_{i}\right]\right] \stackrel{!}{=} 0 \quad \forall j
$$

which means that $\kappa$ is independent of the retention levels $r_{i}$ indicating that no unique solution exists.

A situation with no solution arises if capital $\operatorname{costs} \lambda$ and $\lambda^{*}$ are zero. This leads to

$$
\frac{\partial \phi}{\partial r_{j}}=\kappa \cdot \frac{\partial}{\partial r_{j}} \sum_{i}\left[\mathrm{E}\left[R_{i}\right]-\mathrm{CES}_{\alpha, Z}\left[R_{i}\right]\right] \stackrel{!}{=} 0 \quad \forall j,
$$

i.e. a condition that is generally only fulfilled with $\kappa=0$. Thus both, equal portfolios and zero capital costs lead to a situation where no optimization is possible. Loosely speaking, the reason for this is that in the first case the loadings of reinsurer and primary insurer are proportional. In the second case the loadings itself vanish leaving $\kappa=0$ as a degenerate solution.

\subsubsection{Unconstrained risk retention}

Optimal retentions without constraint related to the overall net risk retention ('absolute retentions') maximize the overall profit

$$
\mathrm{E}[Z]=\max !
$$

leading to a similar criteria as (3.14) but with $\kappa=0$ in $\phi$

$$
\begin{aligned}
\frac{\partial \phi}{\partial r_{j}} & =\frac{\partial}{\partial r_{j}}\left[\left(\lambda^{*}-\lambda\right) \cdot \mathrm{E}\left[R_{j}\right]-\sum_{i}\left[\lambda^{*} \cdot \mathrm{CES}_{\beta, Z^{*}}^{*}\left[R_{i}\right]-\lambda \cdot \mathrm{CES}_{\alpha, Z}\left[R_{i}\right]\right]\right] \\
& =\frac{\partial \phi}{\partial r_{j}} \sum_{i}\left[\lambda \cdot\left(\operatorname{CES}_{\alpha, Z}\left[R_{i}\right]-\mathrm{E}\left[R_{i}\right]\right)-\lambda^{*} \cdot\left(\operatorname{CES}_{\beta, Z^{*}}^{*}\left[R_{i}\right]-\mathrm{E}\left[R_{i}\right]\right)\right] \stackrel{!}{=} 0 \forall j
\end{aligned}
$$


The implicit assumption here is that the primary insurer does not care about the net risk retention level. In our context this means that there is no constraint related to the available risk capital or, respectively, the risk-based capital to be deployed. The extremum characterized by (3.19) is a maximum if the Hesse matrix $\left.\frac{\partial^{2}}{\partial r_{i} \partial r_{j}} \mathrm{E}[Z]\right|_{r=r_{0}}$ is negative definite.

\subsubsection{The situation of the reinsurer}

A special case which is interesting to consider is the situation where capital costs of reinsurer and insurer are the same $\left(\lambda^{*}=\lambda\right)$. Condition (3.19) then is independent of $\lambda$ and becomes

$$
\frac{\partial \phi}{\partial r_{j}}=\frac{\partial}{\partial r_{j}} \sum_{i}\left[\operatorname{CES}_{\alpha, Z}\left[R_{i}\right]-\mathrm{CES}_{\beta, Z^{*}}^{*}\left[R_{i}\right]\right] \stackrel{!}{=} 0 \quad \forall j
$$

Thus we find

$$
\nabla_{r} \mathrm{CES}_{\alpha, Z}[R] \stackrel{!}{=} \nabla_{r} \mathrm{CES}_{\beta, Z^{*}}^{*}[R]
$$

which means that optimal retentions $r_{i}$ are characterized by an equal gradient $\nabla_{r}$ of the conditional Expected Shortfall of the overall recovery $R$ with respect to insurer portfolio $Z$ and the reinsurer portfolio $Z^{*}$, respectively. Note that this condition still depends on portfolio structures and risk appetite levels $\alpha$ and $\beta$.

Relation (3.21) has an interesting interpretation as from its symmetry we can conclude that risk sharing characterized by (3.21) is optimal for both the insurer and the reinsurer. Relation (3.21) also is reminiscent of some results obtained in the context of the theory of cooperative games. As has been shown in [9], the Aumann-Shapley value provides a fair allocation principle that for coherent risk measures simply corresponds to the gradient of the risk measure with respect to presence levels of sub-portfolios ${ }^{7}$. While the setting in [9] describes one firm with sub-portfolios, here two firms are considered that evaluate an allocation of risk-based capital (i.e. capital cost) for transferring risk $R$ via risk transfer instruments. In the case of one firm, risk and diversification is fully shared and the fair risk-based capital allocation corresponds to the gradient of the risk measure with respect to presence levels of sub-portfolios. If two firms share risk via risk transfer instruments, both benefit from sharing diversification only to the limited extent of actually transferred risk $R$. Without additional constraints, the fair and optimal allocation then is characterized by $r_{0}=r$ fulfilling (3.21).

\footnotetext{
7 Note that the allocation principle (2.13) corresponds to the Aumann-Shapley method for homogeneous risk measures and portfolio profit/loss distributions.
} 


\subsection{Reinsurance structures}

Based on the optimality criteria (3.14), this Section presents a brief discussion of optimal retentions for common reinsurance structures and discusses implementation questions. The Section also relates to Section 4, where a numerical example is considered.

We consider here the simplified situation where both proportional and nonproportional reinsurance is characterized by one parameter only. For a non-proportional reinsurance structure on a single claim basis (e.g. excess-of-loss), the parameter is the attachment point above which risk is transferred, or equivalently the deductible. No limit is considered. For a proportional reinsurance agreement, the parameter is the pro-rata amount of risk that is retained. A generalization to to risk transfer characterized by more parameters is straightforward.

\subsubsection{Proportional reinsurance}

Proportional reinsurance leads to recoveries

$$
R_{i}=\left(1-r_{i}\right) \cdot L_{i}=\left(1-r_{i}\right) \cdot \sum_{j=1}^{N_{i}} l_{j, i}
$$

where $r_{i}$ is the proportional risk retention by the primary insurer (per line of business or risk category $i$ ). Inserting this expression in (3.14) leads to the following expression

$$
\begin{aligned}
& \frac{\partial \phi}{\partial r_{j}}=\left(\lambda^{*}-\lambda+\kappa \cdot\left(1-\lambda^{*}\right)\right) \cdot \mathrm{E}\left[L_{j}\right] \\
& -\frac{\partial}{\partial r_{j}}\left[\sum_{i} r_{i} \cdot\left[\lambda^{*} \cdot(1-\kappa) \cdot \operatorname{CES}_{\beta, Z^{*}}^{*}\left[L_{i}\right]-(\lambda-\kappa) \cdot \mathrm{CES}_{\alpha, Z}\left[L_{i}\right]\right]\right] \stackrel{!}{=} 0 \quad \forall j
\end{aligned}
$$

On first sight, this expression may appear to be independent of $r_{i}$. However, this is not the case since the risk measures $\mathrm{CES}_{\alpha, Z}$ and $\mathrm{CES}_{\beta, Z^{*}}^{*}$ by $Z=Z[r]$ and $Z^{*}=Z^{*}[r]$ implicitly depend on $r$. Loosely speaking, the linearity of underwriting result and capital costs under a proportional reinsurance agreement thus is lost once a risk-based commission as implicitly defined by (3.8) is taken into account.

While proportional reinsurance leads to a simplified optimality criteria (3.23), the implicit dependence of risk measures $\mathrm{CES}_{\beta, Z^{*}}^{*}\left[R_{i}\right]$ and $\mathrm{CES}_{\alpha, Z}\left[R_{i}\right]$ on $r_{i}$ remains and a further simplification is not straightforward. In general, a numerical evaluation of (3.23) is necessary.

\subsubsection{Non-proportional reinsurance}

Non-proportional reinsurance on an excess-of-loss basis applies to single losses. In general, the recovery from a reinsurance structure is limited to loss amounts 
between retentions $r_{i}$ and a maximum claim amounts $m_{i}$. As discussed, we consider here only the case where $m_{i}=\infty$ (unlimited layer). The total recovery then is

$$
R_{i}=\sum_{j=1}^{N_{j}} \max \left[l_{i, j}-r_{i}, 0\right]
$$

While finding analytical expressions in terms of the severity and frequency distributions $S_{i}$ and $N_{i}$ for E[ $\left.R_{i}\right]$ is feasible (see e.g. [11]), closed form expressions for $\mathrm{CES}_{\beta, Z^{*}}^{*}\left[R_{i}\right]$ and $\mathrm{CES}_{\alpha, Z}\left[R_{i}\right]$ and corresponding derivatives with respect to retention levels $r_{i}$ are not readily available and may not exit. We thus do not attempt to write (3.14) in terms of severity and frequency distributions for $S_{i}$ and $N_{i}$. Instead, a numerical evaluation will be considered.

\subsubsection{Stop loss reinsurance}

Stop loss reinsurance applies to the aggregated loss amounts. The recoveries for a reinsurance structure with aggregate retentions $r_{i}$ and limits $m_{i}=\infty$ are

$$
R_{i}=\max \left[L_{i}-r_{i}, 0\right]
$$

As is the case with excess-of-loss reinsurance, risk measures $\mathrm{CES}_{\beta, Z^{*}}^{*}\left[R_{i}\right]$ and $\mathrm{CES}_{\alpha, Z}\left[R_{i}\right]$ implicitly depend on $r_{i}$. Finding analytical expressions for derivatives of $\mathrm{CES}_{\beta, Z^{*}}^{*}\left[R_{i}\right]$ and $\mathrm{CES}_{\alpha, Z}\left[R_{i}\right]$ with respect to $r_{i}$ is thus not straightforward.

\subsection{Some remarks}

\subsubsection{Technicalities}

A straightforward way of addressing the risk retention problem numerically are Monte Carlo methods frequently used for Asset Liability Management (ALM) and Dynamic Financial Analysis (DFA). In such a scenario based setting, the Expected Shortfall and its co-measure counterpart, the conditional Expected Shortfall, transform simply into averages over subsets of scenarios. Computing these conditional averages can be done easily and efficiently. From the practical point of view, another important property of risk measures used here is stability. Compared to Value-at-Risk, the Expected Shortfall is a much more stable quantity since it is defined as an average over quantiles instead of a single quantile. This is of special importance when it comes to compute partial derivatives e.g. in (3.14). Numerically, the main problem that has to be tackled in the Monte Carlo setting are discontinuities due to finite resolution in both the objective functions $Z_{i}$ and the constraint $\mathrm{RBC}[Z]$. One option is to continuously approximate or smooth the corresponding functions. In addition, numerical optimization schemes available in advanced mathematical software 
packages such as MATLAB provide a wide range of methods to resolve numerical problems related to discontinuities.

\subsubsection{Practical feasibility and relevance}

In practice, risk transfer takes place without a detailed knowledge of the portfolio structures on the other side of the transaction. In general, portfolio information is passed to the market only indirectly by quoted rates. The concept of 'leading and following reinsurers' implies that rates do not take into account the portfolio structure of a major part of market participants. The current (re-)insurance market thus is not well enough developed in order to fully allow for risk transfer guided by the scheme outlined here. However, approximations based e.g. on simplified portfolio models and considering optimal retentions as first indications to be adjusted by expert judgement later may prove helpful in practice.

The situation is somewhat different when it comes to intercompany risk transfer. Intercompany risk transfer plays an important role for capital management of multinational (re-)insurance groups. Instead of capital, risk is often transfer between balance sheets. Under the assumption that multinational groups have a risk management process and system in place that allows for a quantitative assessment of risk on portfolio and sub-portfolio level, risk transfer guided by principles discussed above will allow the group to use its capital in an efficient way despite the fact that capital might be tight up on individual balance sheets. However, in such a situation also the legal entity and ownership structure (and corresponding risk) need to be taken into account. For an example of optimization of capital requirements within an insurance group see [13].

An important force that will eventually require more transparency from the (re-)insurance industry regarding risk transfer is the regulatory shift towards risk-based supervision. In Europe, Solvency II for instance will impose a riskbased regulatory framework based on prescribe risk measures and confidence level. By this, the framework introduces comparability into the market and paves the way towards a market place that eventually may allow for a more transparent sharing of risk.

\section{An illustrative EXAMPle}

In this Section we present illustrative results of a simple model. The model demonstrates risk transfer from a portfolio consisting of two risk classes to a portfolio that is similar but more diversified. Losses are modeled by frequency and severity distributions. Even this simple example illustrates how diverse effects of risk transfer are and that sharing the 'right' part of the risk has a considerable impact on the economic bottom line.

The example is based on $50^{\prime} 000$ Monte Carlo scenarios. Characteristics of risk classes are listed in Table 1 and risk appetite levels and cost of capital 
TABLE 1

RISK CHARACTERISTICS OF THE RISK CEDING (RISK CLASSES 1A AND 1B) AND THE RISK RECEIVING PORTFOLIO (RISK CLASSES 2A AND 2B). RISK CLASSES ARE INDEPENDENT.

NOTE THAT THE RISK RECEIVING PORTFOLIO IS MORE DIVERSIFIED

\begin{tabular}{l|lccc}
\hline \hline & \multicolumn{2}{|c}{ Severity } & \multicolumn{2}{r}{ Frequency } \\
\hline & Typ & $\mu$ & $\sigma$ & $f$ \\
\hline Risk Class 1a & Normal & 2.0 & 1.0 & 2 \\
Risk Class 1b & LogNormal & 2.0 & 1.5 & 4 \\
Risk Class 2a & Normal & 2.0 & 1.0 & 4 \\
Risk Class 2b & LogNormal & 2.0 & 1.5 & 8 \\
\hline \hline
\end{tabular}

TABLE 2

RISK APPETITE LEVELS AND COST OF CAPITAL FOR RISK TRANSFERRING PORTFOLIO (1) AND RISK RECEIVING PORTFOLIO (2).

\begin{tabular}{l|cc}
\hline \hline & Risk Appetite & Cost of Capital \\
\hline Portfolio 1 & $90 \%$ & $9 \%$ \\
Portfolio 2 & $90 \%$ & $14 \%$ \\
\hline \hline
\end{tabular}

in Table 2. We chose the risk appetite for both portfolios to be the same but consider different capital costs. The first portfolio could be identified with a primary insurer, the second portfolio with a reinsurer. Considering a common risk appetite level is in the spirit of a risk-based supervision framework that does not distinguish between primary insurer and reinsurer. Different capital costs are considered in order to illustrate the dependency on this parameter. Note that for the first, risk ceding portfolio capital costs are lower than the capital costs of the second, risk receiving portfolio. We have chosen this relation because it leads to a situation with an unconstraint maximum for the expected net profit including capital costs $\mathrm{E}[Z]$.

In Figures 1 to 4 we plot optimal retentions, diversification and expected net loss and profits as well as the loading of the reinsurance premium, all as a function of the net risk-based capital. A proportional and a non-proportional excess-of-loss reinsurance agreement is considered. For illustration, we also plot a number of other relations, e.g. between retentions and reinsurance premium loading. Finally we also present surface plots illustrating the behavior of the net result over the whole retention range. Note that this type of figure is based on an 'brut-force' calculation taking into account a large number of possible combinations of retention levels $r_{i}$. For the determination of retention levels these calculations are not used. All Figures are based on the assumption that the primary insurer receives a premium that compensates the expected loss and capital costs as discussed in Section 3.2. 

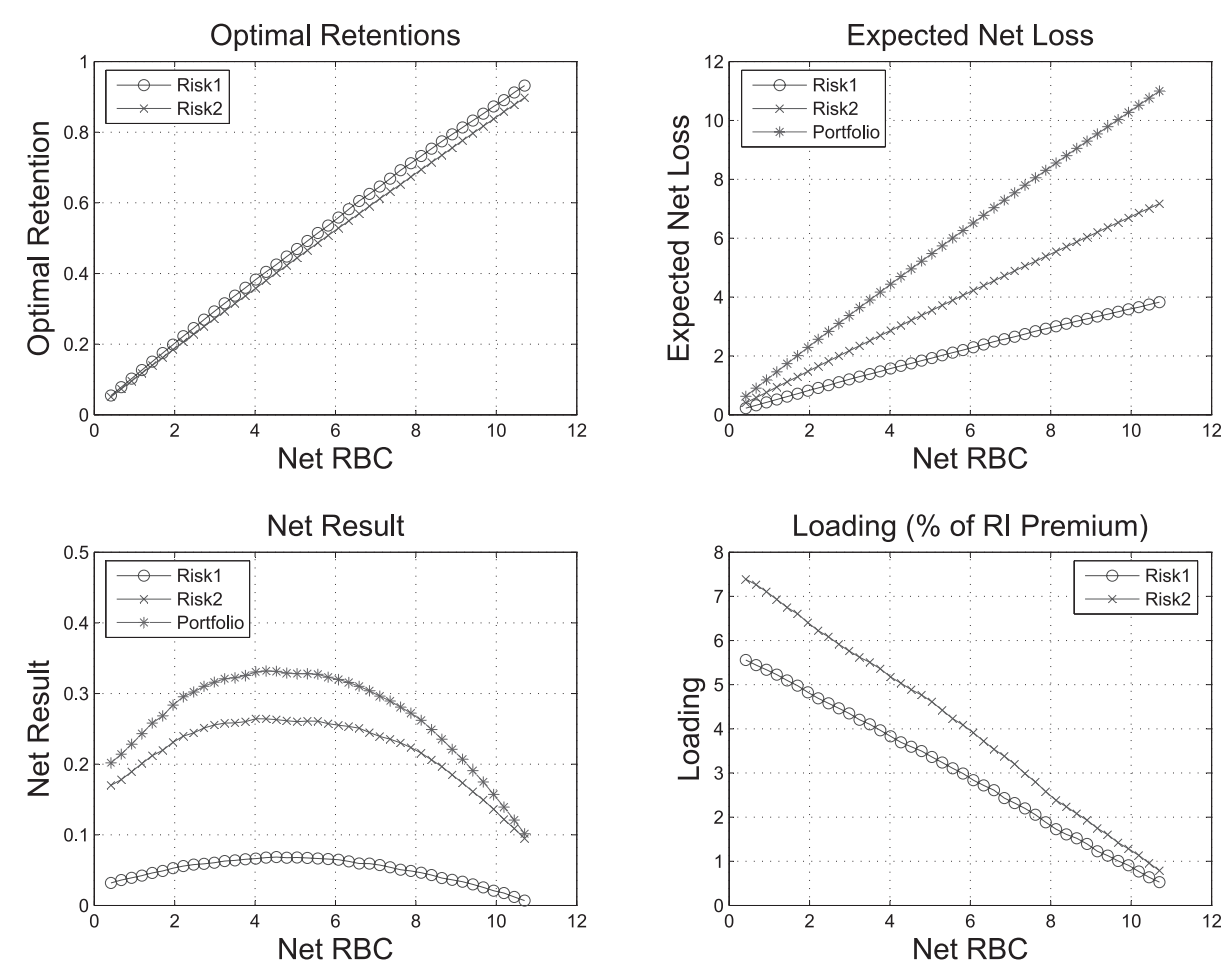

FIgure 1: Proportional reinsurance. Optimal retentions and net result as a function of the total net riskbased capital (left panes). Right panes show the expected net loss (top) and the loading of the reinsurance premium, again as a function of the net risk-based capital.

The quantitative behavior observed in Figures 1 to 4 is understood in terms of factors driving the expected net underwriting result of the primary insurer $Z$ and the factors driving the reinsurance premium $R$. Main drivers are diversification and capital costs. Note for instance that the overall net result is increasing as retentions are lowered. Behind this feature is the fact that the diversification effects tend to increase as a bigger part of the risk is shared. As well, sharing both types of risk turns out to be better than sharing just one type of risk. As can be seen from Figures 1 and 3 as well as Figures 2 and 4, optimal retentions are characterized by ceding similar parts of the risk. This again has to do with diversification effects. Finally note that in both the proportional and non-proportional case there is an absolute maximum of the net result which can be traced back to the assumption that capital costs for the portfolio taking over risk is higher than capital costs of the risk ceding portfolio. The more risk is ceded, the more the higher capital costs of the risk receiving portfolio impact, through the reinsurance premium, the net result. An interesting result of its own is the fact that from an absolute point of view the proportional reinsurance structure is favored over the non-proportional 


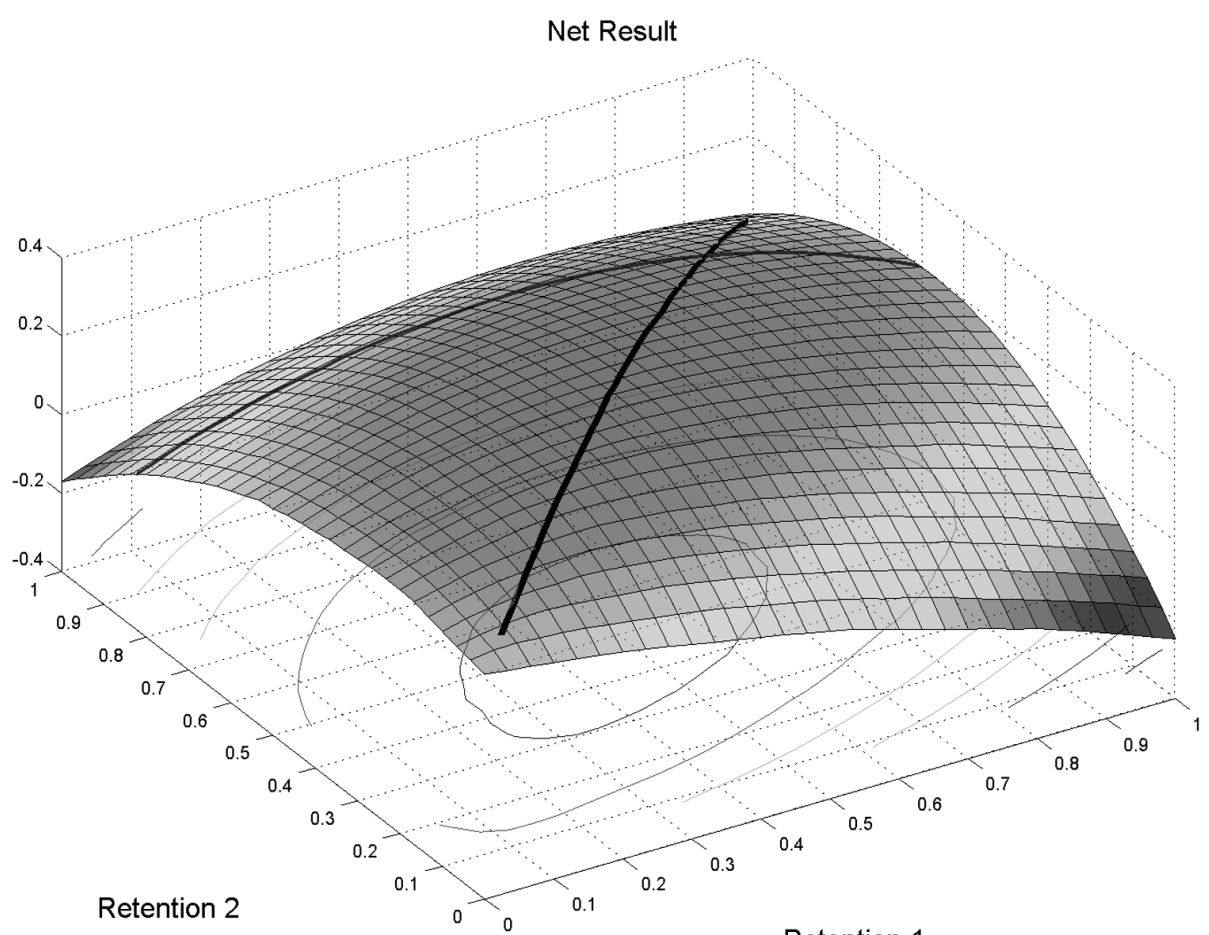

Retention 1

Figure 2: Proportional reinsurance. Net result as a function of retention levels. The dark line indicates optimal retentions as calculated form condition (3.14). The bright line represents the constraint net result at $\mathrm{RBC}=8.5$. It illustrates the range of achievable net results with fixed RBC amount.

one. This can be traced back to diversification effects which tend to be bigger for higher net risk retention levels in the case of the proportional reinsurance structure.

Looking at Figures 3, another important feature becomes apparent. There are situations, where optimal retentions are hard to isolate because another combination of retention levels is nearly as optimal. In this case numerical instabilities introduce noisy fluctuations in retention levels. However note that these fluctuations do not affect the maximization of the overall net result. The noisy fluctuations observed especially in the lower left pane of Figure 3 are thus merely an allocation problem.

When interpreting these results it should be kept in mind that the discussed behavior is specific for the simple model we have chosen. If for example dependency structures are considered either in the risk ceding, the risk receiving or between risk ceding and receiving portfolio, optimal retentions are clearly different. Again, this is due to different diversification effects. A positive dependency between risks in the transferring and receiving portfolio e.g. leads to a corresponding higher reinsurance premium and will thus favor transferring 

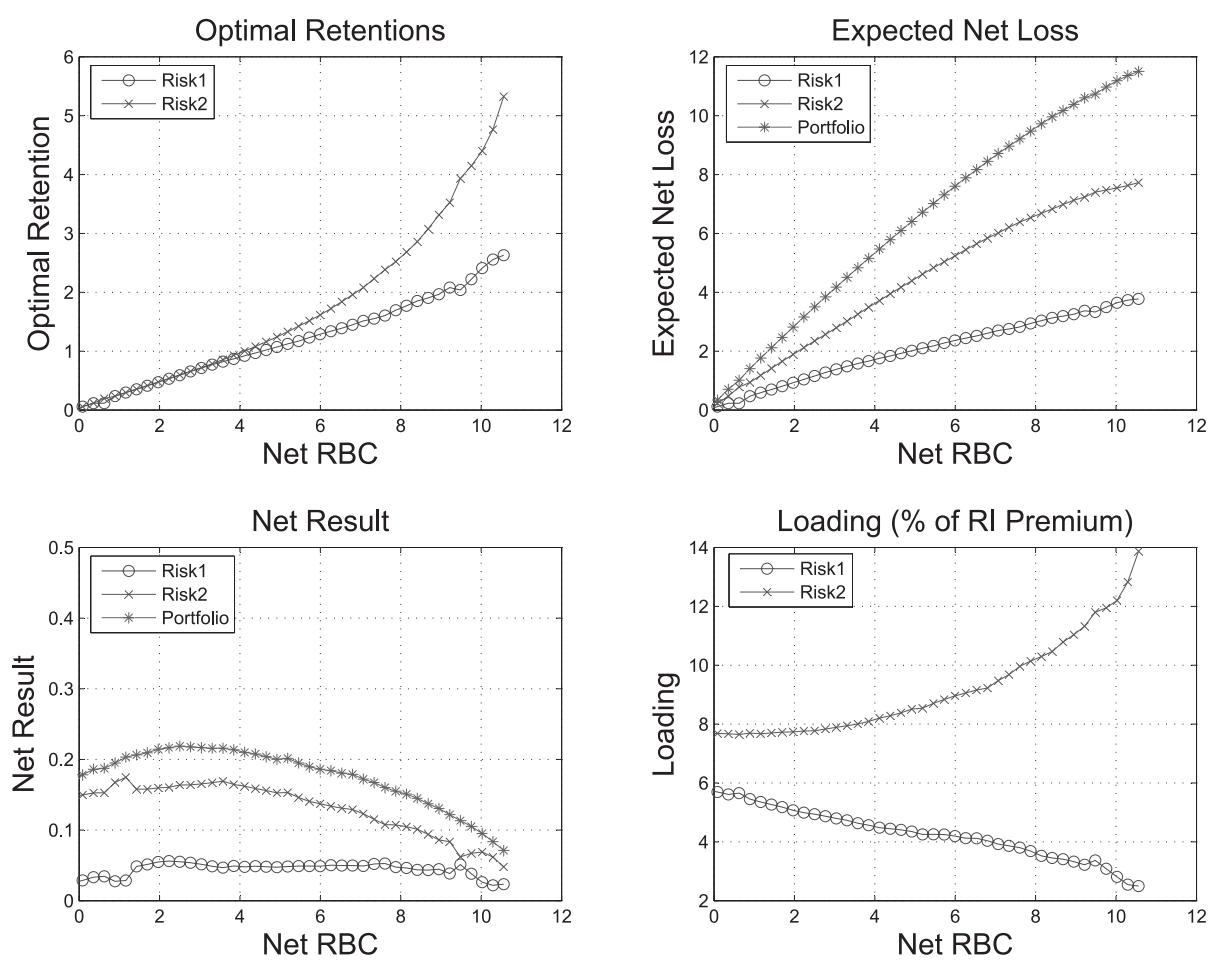

FIGURE 3: Non-proportional excess-of-loss reinsurance. Optimal retentions (or deductibles) and net result as a function of the total net risk-based capital (left panes). Right panes show the expected net loss (top) and the loading of the reinsurance premium, again as a function of the net risk-based capital. Note the noisy behavior of optimal retentions around $\mathrm{RBC}=9$ and $\mathrm{RBC}=1$. While the optimal net result on sub-portfolio level is affected (see lower left pane), the optimal overall net result is not. For a discussion see Section 4.

other, less dependent risks. Clearly, changing the risk characteristics (e.g. severity distribution) will also have an impact on optimal retentions.

\section{SUMMARY AND CONCLUSIONS}

The question of optimal risk transfer has a long history. In terms of minimizing the Variance of the overall net result, the setting of optimal retention levels of reinsurance programs dates back to de Finetti's 1940 paper [10]. In depth research has been performed since then, ranging from approaches relying on criteria related to adjustment coefficients to utility theory. The advent of new actuarial and financial risk management methods including advanced risk measures and Monte Carlo techniques makes it possible to address the problem of optimal risk transfer from yet another point of view.

In this paper we considered the impact of risk transfer on the economic bottom line of an insurer by taking into account capital costs. Capital costs relate 


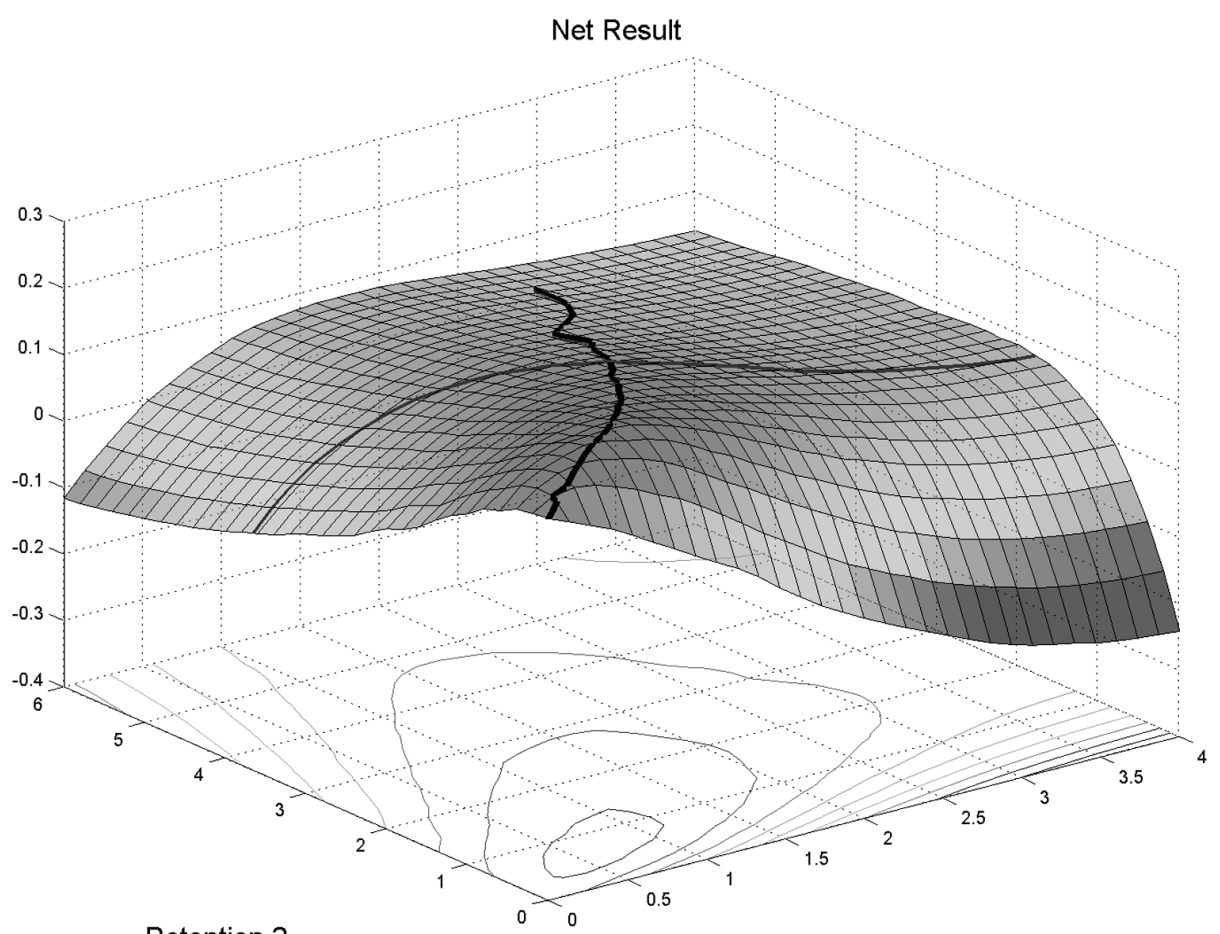

Retention 2

Retention 1

FIGURE 4: Non-proportional excess-of-loss reinsurance. Net result as a function of retention levels (or deductibles). The dark line indicates optimal retentions as calculated form condition (3.14). The bright line represents the constraint net result at $\mathrm{RBC}=8.5$. It illustrates the range of achievable net results with fixed $\mathrm{RBC}$ amount.

to risk-based capital which we define by means of the Expected Shortfall. On sub-portfolio level, capital is allocated conditional to the overall portfolio thus consistently taking into account diversification effects. We assume that for both, the risk ceding and risk receiving portfolio similar capital allocation and risk pricing procedure are used. Starting from these assumptions, we derive a criteria for optimal risk retention levels on sub-portfolio level. Optimal retentions maximize the net result including capital cost given a constraint on available risk-based capital. Implicitly our approach also determines an optimal capital allocation on a lines of business level that depends on overall available capital and the price of risk transfer. In this respect the paper addresses a similar problem as [16]. The crucial question discussed in [16] of how available information with regard to the dependency structure impacts capital allocation warrants a further analysis in our context of optimal risk transfer. Another extension or rather generalization of the presented methodology could be achieved by incorporating elements of utility theory, e.g. by changing the probability measure and computing risk measures with respect to distorted 
probabilities. Further, similar results partly hold for a situation in which the insurer and reinsurer do not use the same risk measure but apply different yet positively homogenous risk measures.

The simple numerical example discussed in Section 4 illustrates a key conclusion of this paper: To be most advantageous from an economic point of view, not only the sharing of risk but also the sharing of diversification needs to be considered when structuring risk transfer. In practice this implies that ceding very specific risks may not be the most efficient way of sharing risk.

\section{ACKNOWLEDGEMENTS}

This paper is a revised version of a paper originally presented at the ASTIN 2005 conference. The author wishes to thank an anonymous referee and his former colleagues Michel Dacorogna and Christoph Hummel at Converium (now Scor) for valuable comments.

\section{REFERENCES}

[1] Acerbi, C. and TASChe, D. (2002) On the coherence of Expected Shortfall, Journal of Banking and Finance 26, 1487-1503.

[2] Artzner, P., Delbaen, F., Eber, J.M. and Heath, D. (1999) Coherent measures of risk, Mathematical Finance 9, 203-228.

[3] Bühlmann, H. (1970) Mathematical Methods in Risk Theory, Springer-Verlag, Berlin.

[4] Centeno, M.L. (1985) On combining quota quota-share and excess of loss, ASTIN Bulletin 15, 49-63.

[5] Centeno, M.L. (1986) Measuring the effects of reinsurance by the adjustment coefficient, Insurance: Mathematics and Economics 5, 169-182.

[6] Centeno, M.L. (1988) The expected utility applied to reinsurance, in: Risk, Decision and Rationality. Edited by Bertrand R., Munier D., Reidel Publishing Company.

[7] Centeno, M.L. (2002) Measuring the effects of reinsurance by the adjustment coefficient in the Sparre Andersen Model, Insurance: Mathematics and Economics 30, 37-49.

[8] Centeno, M.L. (2004) Retention and Reinsurance Programmes. Encyclopedia of Actuarial Science 3, 1443-1452, Wiley.

[9] Denault, M. (2001) Coherent allocation of risk capital, Journal of Risk 4, 1-33.

[10] DE FinetTI, B. (1940) Il problema dei pieni, Giornale dell'Istituto Italiano degli Attuari 11, $1-88$.

[11] Daykin, C., Pentikänen, T. and Pesonen, M. (1996) Practical risk theory for actuaries, Chapmen \& Hall, New York.

[12] European Commission (2007) Framework Directive for Solvency II (draft), available at http://eur-lex.europa.eu/LexUriServ/site/en/com/2007/com2007_0361en01.pdf

[13] Filipović, D. and KupPeR, M. (2007) On the Group Level Swiss Solvency Test, Mitteilungen der Schweizer Aktuarvereinigung 1, 97-114.

[14] Kalkbrener, M. (2005) An Axiomatic Approach to Capital Allocation, Mathematical Finance 15, 425-437.

[15] Kaluszka, M. (2001) Optimal reinsurance under mean-variance premium principles, Insurance: Mathematics and Economics 28, 61-67.

[16] Laeven, R. and Goovaerts, M. (2004) An optimization approach to the dynamic allocation of economic capital, Insurance: Mathematics and Economics 35, 299-319.

[17] Mildenhall, S.J. (2004) A note on the Myers and Read capital allocation formula, North American Actuarial Journal 8, 32-44. 
[18] Rockafellar, R.T. and Uryasev, S. (2002) Conditional Value-at-Risk for general loss distributions, Journal of Banking and Finance 26, 1443-1471.

[19] Schmitter, H. (2000) Setting optimal reinsurance retentions, SwissRe Publication.

[20] SChNiePer, R. (2000) Portfolio Optimization, ASTIN Bulletin 30, 195-248.

[21] Simon, C.P. and Blume L.E. (1994) Mathematics for Economists, 448-465, W.W. Norton.

[22] Swiss Federal Office of Private Insurance (2004) White Paper of the Swiss Solvency Test.

[23] VAJDA, S. (1962) Minimum variance reinsurance, ASTIN Bulletin 2, 257-260.

[24] TASCHE, D. (2000) Risk contributions and performance measurement, Working Paper, Technische Universität, München.

[25] Venter, G.G. (2004) Capital allocation survey with commentary, North American Actuarial Journal 8, 96-107.

[26] Waters, H. (1979) Excess of loss reinsurance limits, Scandinavian Actuarial Journal, 37-43.

\author{
ANDREAS KULL \\ AXA Winterthur \\ P.O. Box 357 \\ CH-8401 Winterthur, Switzerland \\ E-Mail: andreas.kull@axa-winterthur.ch
}

